\title{
An optimal and adaptive double threshold-based approach to minimize error probability for spectrum sensing at low SNR regime
}

\author{
Garima Mahendru $^{1}$ (1) Anil K. Shukla ${ }^{1} \cdot$ L. M. Patnaik ${ }^{2}$
}

Received: 5 May 2020 / Accepted: 3 November 2021 / Published online: 30 November 2021

(c) The Author(s), under exclusive licence to Springer-Verlag GmbH Germany, part of Springer Nature 2021

\begin{abstract}
With the recent explosion in the number of wireless communication technologies, the frequency spectrum has become a scarce resource. The need of the hour is an efficient method to utilize the existing spectrum and Cognitive Radio is one such technology that can mitigate the spectrum scarcity. In a cognitive radio system, the unlicensed secondary user accesses the spectrum allotted to licensed primary users when it lies vacant. To implement dynamic or opportunistic access of spectrum, secondary users perform spectrum sensing, which is a quintessential part of a Cognitive radio. From the Cognitive user's point of view, lesser error probability means an increased likelihood of channel reuse when it is vacant, and a higher detection probability signifies better protection to the licensed users. In both cases the decision threshold plays a pivotal role in determining the fate of the unused spectrum. In this paper, we study the difficulty of selecting an appropriate threshold to minimize the error probability in an uncertain low SNR regime. The sensing failure issue is analyzed, and an optimal threshold is computed that yields minimum error rate. An adaptive double threshold concept has been proposed to make the detection robust and a closed-form equation for optimal threshold has been derived to minimize the error. The novel findings through simulation results exhibit improvement in Probability of detection and reduction in probability of error at low SNR in the presence of noise uncertainty factor.
\end{abstract}

Keywords Spectrum sensing · Probability of detection · Sample complexity · Adaptive threshold · Optimal threshold · Error probability

\section{Introduction}

The demand to substantiate the access to electromagnetic radio spectrum is at a crossroad. The emerging and ever increasing wireless technological options are attracting more and more demand for spectrum. There has been a significant surge in the demand for more spectrum since the onset of the pandemic due to outbreak of COVID 19 worldwide as everyone is forced to stay home and be online always (Lutu et al.

Garima Mahendru

gmahendru@amity.edu

Anil K. Shukla

akshukla2@amity.edu

L. M. Patnaik

lalitblr@gmail.com

Amity University Uttar Pradesh, Noida, India

2 National Institute of Advanced Studies, IISc Campus, Bangalore, India
2020). Overcrowding of the spectrum seems like a cliché where it is assumed that it is overutilized but on the contrary it has been identified that most of the spectrum is underutilized and that actual cause of the glitch is the existing inefficient scheme of spectral allocation (Tech. Rep. 2002; Valenta et al. 2010) The present spectrum access method is grounded on the principle that segments the spectrum into exclusive licensed and unlicensed frequency bands. The measurements of spectrum occupancy depict that a significant portion of spectrum lies unused in frequency, time and space (McHenry 2005). Cognitive Radio is a concept that promises to alleviate the spectrum shortage by effective and dynamic utilization of electromagnetic spectrum (Mitola and Maguire 1999). To allow dynamic access to the frequency spectrum, secondary users perform spectrum sensing. Spectrum sensing is thus a vital part of a Cognitive radio network which involves detection of an unused spectrum space to setup a communication link for the Secondary User (SU) without disrupting the Primary User (PU) transmission (Haykin 2005). The development of the first 
wireless standard based on Cognitive radio concept IEEE 802.22 form the basis as well as the foundation for ongoing research in the promising area of CRs (Cordeiro et al. 2006). The IEEE 802.22 WRAN standard was intended to function in the TV (Television) broadcast bands and makes sure that the incumbent operation of TV broadcasting, and low power licensed devices are not affected by any harmful interference.

Energy detection (ED) based spectrum sensing method is one of the most explored schemes which still has a lot of room for improvement especially due to its degraded performance at low SNR. It allows the detection of unknown signals without any a priori information with least computational and implementation complexity. (Shukla et al. 2016; Arjoune and Kaabouch 2019; Bhowmick et al, 2015). This work emphasizes on the enhancement of the energy detection scheme by minimizing the error rate (also termed as probability of error) that creeps in at low (signal-to-noise-ratio) SNR in presence of uncertainty. At low $\mathrm{SNR}$, the PU signal is too weak to be detected by the SU which may raise the false alarm. Therefore, low SNR regime is critically very important for spectrum sensing techniques in Cognitive radio systems to reliably detect the incumbent PU signals and increase the detection probability. Furthermore, this paper proposes a double threshold-based spectrum sensing with a dynamic threshold factor to lessen the width of "no decision" region that is caused when the uncertainty in noise exceeds the SNR. The decision threshold is then optimized for this region with minimization of the error probability as the objective function. The performance of the proposed double threshold-based energy detection sensing has been examined thoroughly in terms of false alarm rate and detection probability under low SNR conditions. Comprehensive performance evaluation of energybased detection is provided which depicts enhancement in the detection performance and further minimization of the error rate at an optimal threshold.

The remaining of the paper is organized as follows: Sect. 1 present a brief literature review of the recent research done in the field of spectrum sensing using energy detection technique at low SNR with uncertainty in noise and the outline of the contribution of this paper. Numerous references have been cited to bring out the significance of the proposed work and its relevance to the scientific community. Section 2 gives the general spectrum sensing system model and Sect. 3 presents the proposed method of sensing the spectrum. Section 4 gives the simulation results and discussion with highlighted enhancement and improvement in the detection performance.

\section{Literature review}

Recent research in the spectrum sensing has introduced methods like matched filter detection, cyclo-stationary feature detection, energy detection, cooperative sensing, etc. (Vadivelu et al. 2014; Hosseini et al. 2010; Letaief and Zhang 2009). Matched filter detection and cyclo-stationary based detection offers robust sensing performance but renders increased implementation complexity. The work proposed by authors by Vadivelu et al. (2014), Hosseini et al. (2010), Letaief and Zhang (2009) are conventional individual based spectrum sensing without the use of cooperative communication. Cooperative sensing method also promises enhanced sensing performance which is more reliable but at the cost of increased latency as well as hardware complexity. Extra hardware cost is due to a greater number of sensors deployed for cooperative fusion of a common decision which also adds in some delay in reporting an availability of the spectrum. The difference between a cooperative sensing technique and a non-cooperative sensing method is that the former incorporates a fusion based decision through the concept of cooperative communication (Anandakumar and Umamaheswari 2017). The work presented in this paper emphasizes on the enhancement of a non-cooperative based spectrum sensing method using double adaptive threshold and optimization of decision threshold in the region of uncertainty at low SNR. As discussed in the previous section, energy detection technique which is individual based sensing method without the use of cooperation from more than one detector decoys the attention of the researchers worldwide but is limited by low SNR with uncertain noise. The main reason behind this issue is the pre-defined fixed threshold assignment policy adopted by conventional energy detection method. Various methods have been proposed in the existing literature to combat this problem through an adaptive threshold, double threshold concept or a multi staged detection process. Apart from the methods that attain enhanced detection probability and reduced error rates through dynamic selection of threshold, there exists another major issue that limits the sensing performance. The minimum SNR value termed as "SNR Wall" in Tandra and Sahai (2008) which has been defined as a fundamental limit to SNR for spectrum sensing. It states that with uncertain noise, sensing fails below "SNR Wall" even when detection duration is mounted to a very high extent. The SNR wall for signal detection is related to uncertainty in noise and is calibrated as a major drawback for energy detection method.

The SNR wall has been taken up differently by different authors but fundamentally it has been regarded as result of uncertainty in noise. In Tandra and Sahai (2008), the SNR wall has been defined as the total uncertainty in noise which restricts any sensing method from detection and results in a detection failure. In Alink et al. (2011), the SNR wall has been quantified for cross-correlation that depicts it as a linear function of the amount of noise correlation. The authors in Mariani et al. (2011), have performed an asymptotic analysis of the estimated noise 
power energy detector to compute the criterion for the existence of the SNR wall phenomenon.

The use of double thresholds in spectrum sensing has attracted a lot of attention due its enhanced sensing performance and low complexity. However, this comes at the expense of some sensing performance loss as discussed in Xie and Chen (2012) in which maximum and minimum sensing threshold has been re-evaluated and presented. Adaptive double threshold-based detection given in Zhu et al. (2020) has included a weighting factor as a performance metrics to improve the overall performance. In Ling et al. (2012) an adaptive threshold control is proposed by restricting the average interference to the PU within a target level and computes the threshold as a linear increasing function of the SINR. The authors in Sobron et al. (2015), have presented the use of a cost function dependent on a single parameter which conveys the overall information about the availability of primary users. Another paper proposes a fast two step energy detection-based detection by improving the sampling process of conventional energy detection and further optimizes them (Lai et al. 2015). Some methods further emphasize on the "no decision region" or the "sensing failure" scenario in a double threshold-based sensing. The authors in Avila and Thenmozhi (2015) have proposed a method to overcome sensing failure problem in double threshold-based detection. The work by authors Avila and Thenmozhi (2015) is inspiring and worth mentioning. It not only computes two levels of threshold for enhanced performance under noise uncertainty but also allows its dynamic allocation through a closed form expression. Furthermore, the system has been made better via multiple energy detectors at the receiver end. This work has been taken as the basis for further exploration in Sect. 4.

Apart from adaptive and double threshold-based detection, further improvement in the sensing performance is achieved through optimization. A lot of the existing optimization techniques focus on the cooperative sensing methods via energy detection (Verma and Singh 2015; Na 2017; Mamisi and Sundru 2018; Mabrook et al. 2020, Suneel and Shiyamala 2021). A method based on the cooperative spectrum sensing where each CR incorporates and improved energy detector with multiple antennas for Rayleigh fading channels (Verma and Singh 2015). The authors in $\mathrm{Na}$ (2017) have given an adaptive Neuro-Fuzzy interference system (ANFIS) algorithm for making to detect the optimal and accurate free channels under fading and shadowing conditions. Similarly, in Mamisi and Sundru (2018) the power of all the symbols collected near the spectrum is optimized using peak detection technique which reduces the power caused due to unwanted or noisy data. In some optimization problems for spectrum sensing, the optimal number of sensing nodes is selected to enhance the energy efficiency (Mabrook et al. 2020). That means that the objective is taken to be energy efficiency and the parameter that is optimized is number of sensing nodes in a cooperative sensing environment. The second type of optimization problem attempts to maximize the throughput while maintaining the interference level from PU and other parameters at an acceptable level (Bagheri et al. 2020). The Gaussian model finds recognition in parameter optimization problems to attain minimum error probability. The aim for such methods is to attain minimum error rate or maximum throughput at an optimal threshold or allocated power (Avila and Thenmozhi 2015). The authors in Attpattu et al. (2011) have outlined the significance of adaptive threshold and proposed to enhance the sensing performance in terms of increased detection probability with the use of adaptive threshold that is dependent on the noise variance. The results in Attpattu et al. (2011) after using adaptive threshold has further been optimized using peak detection method.

From the given discussion one can infer that a double threshold-based concept, two thresholds are computed for two different ranges of SNR, while for adaptive one, the threshold is varied in accordance with the SNR. The significance of this work in context to the use of double adaptive thresholds with respect to the recent research has been highlighted in the next section.

\subsection{Contribution}

In above discussion, different methods of representing and interpreting the SNR wall has been given but none of the discussed methods address the "no decision" or region of ambiguity that arises due the SNR threshold. Overall, the existing literature talks about the existence of "SNR Wall", that acts as a constraint for any type of spectrum sensing. Moreover, the schemes available using double threshold, adaptive threshold and dynamic thresholds are devoted to alleviating the degrading effect of noise uncertainty at low SNR due to fixed thresholding. However, only few of them addresses the problem of sensing failure or no decision region that occurs when detection is performed at an SNR lower than the SNR wall. This work takes up the existence of SNR wall and the no decision region at low SNR with uncertain noise. In this paper an attempt has been made to deduce an approach towards adaptive and dynamic double thresholds to combat the sensing failure and enhance the detection capability of an energy detector. Firstly, a concept of double threshold classifies the given sensing problem into two regions of low and high SNR. This helps in targeting the low SNR regime with more precision. As observed in the recent work discussed in the previous section, a variable threshold would fetch better detection probability in presence of uncertain noise. A noise uncertainty factor has been included in the noise model and the adaptive- threshold solely dependent on the noise variance has been derived 
through the proposed closed form equation in this paper. The subsequent sections and the simulated results represent the enhancement in the sensing performance that has been achieved through increased detection probability and reduced error probability at low SNR with uncertain noise using the proposed scheme of adaptive double thresholding.

Furthermore, one major research gap in all discussed optimization problems in Sect. 1 is that noise uncertainty has not been considered and the no decision region below the SNR wall has not been accounted for. This paper hereby takes optimization of the decision threshold with a different approach. Firstly, the sensing performance is made robust using the proposed novel adaptive double thresholds and then closed form expression has been derived for detection threshold at low SNR in presence of noise uncertainty. The results show the comparison of detection performance of the proposed method with the existing energy detection-based methods. The outcomes exhibit a significant improvement in detection probability of $27-39 \%$ and subsequent reduction in error probability at low $\mathrm{SNR}=-10 \mathrm{~dB}$ by using the novel method of spectrum sensing.

\section{General spectrum sensing model}

The energy detector is the most suitable method to sense the existence of a PU, in the nonexistence of complete knowledge of the received signal. It encompasses measurement of the energy in the received signal over a specific time span. For practical reasons, it is assumed that noise follows a Gaussian distribution with a flat band-limited power density spectrum. To approximate the received energy over a fixed time sample of the noise, the sum of squares of statistically independent random variables having zero mean and equal variances is obtained through sampling (Attpattu et al. 2011). The fundamental concept of energy detector relies on the estimate of the energy $\mathrm{E}(\mathrm{X})$ of the received signal to deduce a hypothesis for detection $\left(\mathrm{H}_{0}\right.$ and $\left.\mathrm{H}_{1}\right)$ by each cognitive radio user as described in Fig. 1 and represented by Eq. (1).

$\left\{\begin{array}{l}H_{0}: X(n)=N(n) \\ H_{1}: X(n)=N(n)+S(n)\end{array}\right\}$

In the Eq. (1), X(n) represents the received signal, n(n) represents the noise which is assumed to be white Gaussian and $\mathrm{S}(\mathrm{n})$ is the primary signal. If the spectrum is occupied by a Primary User (PU) signal S(n), the received signal information $\mathrm{X}(\mathrm{n})$ confirms unavailability of the spectrum hole by testing $\mathrm{H}_{1}$. Similarly, if the received signal consists of only noise, it tests the presence of a spectrum hole or availability by testing hypothesis $\mathrm{H}_{0}$.

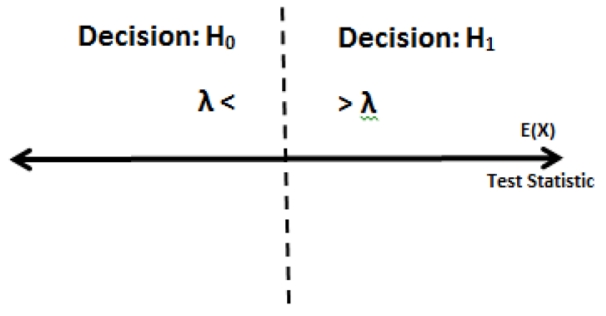

Fig. 1 Energy Detection

The spectrum availability is identified by evaluating the estimated energy to a predefined threshold $\lambda$. The threshold is computed through assumed noise variance and targeted probability of false alarm.

The two-performance metrics for a spectrum sensing technique are detection Probability $\left(\mathrm{P}_{\mathrm{D}}\right)$ and probability of false alarm $\left(\mathrm{P}_{\mathrm{FA}}\right)$. Detection probability is described as the probability that the detector can effectively detect the existence of PU whereas Probability of False Alarm $\left(\mathrm{P}_{\mathrm{FA}}\right)$ is the probability that the detection method declares the presence of the primary falsely due to noise. The mathematical expression for detection probability $\left(\mathrm{P}_{\mathrm{D}}\right)$ and the false alarm probability $\left(\mathrm{P}_{\mathrm{FA}}\right)$ is given as:

$P_{F A}=Q\left(\left(\frac{\lambda}{N \sigma_{n}^{2}}-1\right) \sqrt{N}\right)$

$P_{D}=Q\left(\left(\frac{\lambda}{N \sigma_{n}^{2}}-1-S N R\right) \sqrt{\frac{N}{1+2 S N R}}\right)$

where $Q($.) denotes Gaussian Complementary Cumulative Distribution Function. In these expressions, $\mathrm{N}$ denotes the number of samples which may be expressed as sample complexity $\left(10 \log _{10} N\right)$ (Urkowitz 1967]. The decision threshold $\lambda$ and the number of samples $\mathrm{N}$ are major factor which ultimately decides the vacant spectrum allocation to the $\mathrm{SU}$ and is expressed mathematically as in (4) and (5) according to Urkowitz (1967):

$\lambda=N \sigma_{n}^{2}+Q^{-1}\left(P_{F A}\right) \sqrt{2 N \sigma_{n}^{2}}$

$N=\frac{2\left[Q^{-1}\left(P_{F A}\right)-Q^{-1}\left(P_{D}\right)(1+S N R)\right]^{2}}{S N R^{2}}$

It can be observed from Eqs. (5) that if the SNR becomes low, then the number of samples $(\mathrm{N})$ would increase drastically that will make sensing impractical to implement. Furthermore, if the uncertainty in noise $(\rho)$ is introduced for practical purposes and reliable performance, the Eqs. 2 and 3 would be modified to following expressions such that noise variance $\sigma_{n}{ }^{2}$ 
lies in the interval $\left[\sigma_{n}^{2} / \rho, \rho \sigma_{n}^{2}\right]$ with $\rho \geq 1$ as presented in $\mathrm{Yu}$ et al. (2012).

$$
\begin{aligned}
& P_{F A}=Q\left(\left(\frac{\lambda}{N \sigma_{n}^{2} \rho}-1\right) \sqrt{N}\right) \\
& P_{D}=Q\left(\left(\frac{\lambda}{N \sigma_{n}^{2} \rho}-1-S N R\right) \sqrt{\frac{N}{1+2 S N R}}\right)
\end{aligned}
$$

At low SNR and uncertain noise environment the selection of detection threshold $(\lambda)$ by each SU becomes a crucial task to avoid missed detection or raised false alarm. In such a scenario sensing capability of the detection scheme becomes doubtful. It leads to a sensing malfunction illustrated by ambiguous/ uncertainty zone as shown in Fig. 2 even at higher sensing time (or greater number of samples $\mathrm{N}$ )

Because of the fluctuating noise, the received signal cannot be differentiated from noise and leads to higher error rate even at higher values of $\mathrm{N}$ or sensing time $\left(\tau=N f_{s}\right)$. The error rate is defined as the error probability which is the sum of false alarm and missed detection probability. $\left(\mathrm{P}_{\mathrm{E}}=\mathrm{P}_{\mathrm{FA}}+\mathrm{P}_{\mathrm{MD}}\right)$. If $\rho=1$, there exists no noise uncertainty and the situation is like an ideal case as expressed in (2) and (3). It can be observed that the noise uncertainty factor tries to increase the $\mathrm{P}_{\mathrm{FA}}$ and reduce the $P_{D}$ thereby effecting the system performance in an adverse manner. To compute the number of samples $\mathrm{N}$, the Eqs. (6) and (7) are used and the detection threshold is equated as shown in Eq. (8).

$$
N=\frac{2\left[\rho Q^{-1}\left(P_{F A}\right)-Q^{-1}\left(P_{D}\right)(1 / \rho+S N R)\right]^{2}}{\left(S N R-\frac{(\rho-1)}{\rho}\right)^{2}}
$$

Upon investigating the above-mentioned expression (8), one can infer the influence of uncertain noise on sensing in context to number of samples, detection duration and detection sensitivity. The number of samples $\mathrm{N}$ is basically the length of the samples over which the received signal is averaged to obtain its energy and thus, for better sensing performance it should not increase infinitesimally.

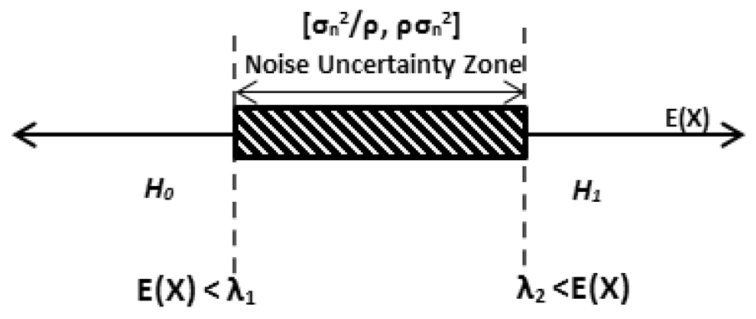

Fig. 2 Region of Uncertainty/Confusion
The consequence of including the uncertainty in noise is that the sensing performance deteriorates with increasing uncertainty factor according abovementioned Eqs. (6) and (7) which is further depicted in the simulation results.

At low SNR and in presence of noise uncertainty $\rho$, the sample number $N \rightarrow \infty$, as $\mathrm{SNR}<<1$. The sharp increase in $\mathrm{N}$ at low SNR and $\rho>1$ is defined as sensing failure which is major tradeoff to sensing problems. The "SNR wall" is quantified as the total uncertainty in noise and is expressed as $\rho-\frac{1}{\rho}$ and is taken as a major constraint that leads to failure of the detection capability. To attain enhanced sensing performance and ease the adverse impact of noise uncertainty, dynamic threshold factor $\rho^{\prime}$ is introduced in (2) and (3) in such a way that $\lambda$ lies in the interval $\left[\lambda / \rho^{\prime}, \lambda \rho^{\prime}\right]$ as discussed in Madhunala and Rallapalli (2020), Attpattu et al. (2011). The sample number $\mathrm{N}$ is now written as:

$$
N=\frac{2\left[\rho{\overline{\rho^{\prime} Q}}^{-1}\left(P_{F A}\right)-\rho^{\prime}\left(\frac{1}{\rho}+S N R\right) Q^{-1}\left(P_{D}\right)\right]^{2}}{\left[S N R+\frac{\rho^{\prime}}{\rho}-\frac{\rho}{\rho^{\prime}}\right]^{2}}
$$

The Eq. (9) for $\mathrm{N}$ clearly depicts that a dynamic threshold factor is introduced to annul the degrading effect of $\rho$ on detection probability. In absence of $\rho^{\prime}$ and presence of $\rho$, the sample number $N \rightarrow \infty$ when SNR is low (SNR $<<1)$. The sharp increase in $\mathrm{N}$ at low SNR and $\rho>1$ is defined as sensing failure. Therefore, an Energy Detector based on Double Threshold concept (ED_DT) gives reliable results and reduces the effect of noise fluctuations. The two thresholds $\lambda_{1}, \lambda_{2}$, incorporate the uncertainty factor to account for the degradation in performance caused by it and are expressed as follows according to Verma and Singh $(2015,2016)$ :

$\lambda_{1}=(\rho-1) \lambda$

$\lambda_{2}=(\rho+1) \lambda$

However, the above expressions do not account for the detection in the region of confusion. The method described in Verma and Singh (2015) makes use of cooperative sensing to generate two-bit decisions to detect the signals that lie in the range $\left(\lambda_{1}<\mathrm{E}(\mathrm{X})<\lambda_{2}\right)$. It increases the complexity and the cost involved in incorporating a fusion decision center.

\section{Proposed model}

In this section, the proposed model for spectrum sensing using adaptive double threshold concept has been presented. The case when the received energy lies in the region of confusion between the two thresholds at low SNR is considered and a method with adaptive double threshold (ED_ADT) is 
proposed. Further with optimal threshold in the uncertainty zone, a minimum probability of error is achieved for minimum error during sensing.

\subsection{Adaptive double threshold (ED_ADT)}

The double threshold concept (ED_DT) discussed in Sect. 3 classifies the test statistic $\mathrm{E}(\mathrm{X})$ in:-State 1: PU signal present $\left(\mathrm{E}(\mathrm{X})>\lambda_{2}\right.$; State 0: only noise present $\left(\mathrm{E}(\mathrm{X})<\lambda_{1}\right)$ and State $x$ : Confused $\left(\lambda_{1}<\mathrm{E}(\mathrm{X})<\lambda_{2}\right)$. Based on the positive influence of dynamic threshold factor $\rho$ ', this section proposes a novel method of spectrum sensing based on computation of two adaptive thresholds in the uncertainty zone (ED_ADT). The confused region $\left(\lambda_{1}<\mathrm{E}(\mathrm{X})<\lambda_{2}\right)$ is taken into consideration and is further sub-divided by two adaptive/dynamic thresholds $\lambda_{10}$ and $\lambda_{20}$ as shown in Fig. 3.

The thresholds $\lambda_{10}$ and $\lambda_{20}$ are derived from Eq. (5) and (6) and a dynamic threshold factor is introduced in such a way that they lie between $\lambda_{1}$ and $\lambda_{2}$ respectively. The dynamic threshold factor $\rho$ ' increases $\lambda_{1}$ to $\lambda_{10}$ and reduces $\lambda_{2}$ to $\lambda_{20}$ as expressed below:

$\lambda_{10}=(\rho-1) \rho^{\prime} \lambda$

$\lambda_{20}=\frac{(\rho+1)}{\rho^{\prime}} \lambda$

According to Eqs. (12) and (13), when the sensing is carried out in the confused state then the decision is made more reliable by sensing the energy again and comparing it to the newly set thresholds. The inclusion of the dynamic threshold factor aims at canceling out the adverse influence of the noise uncertainty factor. After the two adaptive thresholds have been generated according proposed ED_ADT, the decision $\mathrm{D}$ in the sensing process involves following rule:

$D=\left\{\begin{array}{l}1 E(X)>_{20} \\ x_{10}<E(X)<_{20} \\ 0 E(X)<_{10}\end{array}\right\}$

The two novel thresholds $\lambda_{10}$ and $\lambda_{20}$ are termed as "adaptive" as they depend on the variable uncertain noise

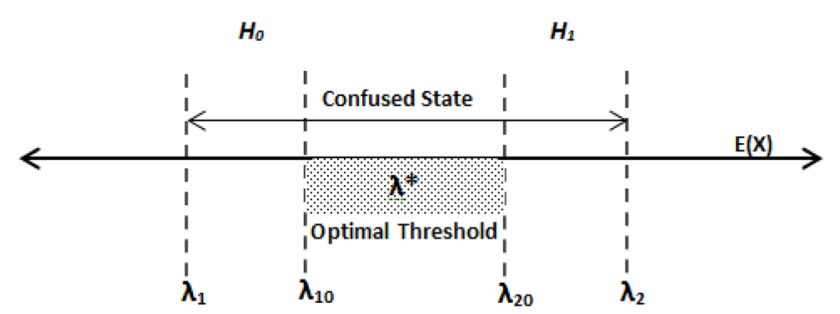

Fig. 3 Dynamic double threshold concept $(\rho)$ and a dynamically changing parameter $\left(\rho^{\prime}\right)$. Apart from these two factors, it is dependent on $\lambda$ of Eq. (4) which is in turn dependent on noise variance $\sigma_{n}^{2}$ ED_ADT displays improved detection probability when $\rho^{\prime}>\rho$ such that the boundary of uncertainty zone shrinks (in Fig. 3). The main aim is to lower the false alarm and raise detection probability at low SNR to increase system robustness. The proposed ED_ADT works well at smaller values of SNR and promises enhanced detection performance even with increasing uncertainty in noise as confirmed by the simulation results in Sect. 4.

\subsection{Optimal threshold for uncertainty zone}

In the previous sub-section, novel approach based on adaptive double threshold for energy detector method for spectrum sensing was proposed to increase the robustness of the system. As per the proposed model in Eqs. (12) and (13), the double thresholds are made adaptive solely to make the sensing decision more reliable and robust, when the detection is carried out in the confused state. The inclusion of the dynamic threshold factor aims at canceling out the adverse influence of the noise uncertainty factor. However, the problem of confusion or no decision region persists if the measured energy is within the range $\lambda_{10}<\mathrm{E}(\mathrm{X})<\lambda_{20}$. After the two adaptive thresholds have been generated according proposed method, the decision $\mathrm{D}$ in the sensing process follows the rule as per Eq. (14) of Sect. 4.1. When the received energy lies within the uncertainty zone, sensing failure occurs and cannot be completed even if sensing duration is lengthened. This sensing failure issue can be addressed with the help of an optimal detection threshold. To sense the spectrum in the confused state and to attain minimum error probability in this region, an optimal threshold value $\lambda^{*}$ is deduced based on the Gaussian model for parameter optimization (Avila and Thenmozhi 2015). It has been observed in conventional energy detection with single threshold (ED), with double threshold (ED_DT) and adaptive double threshold (ED_ADT) concept that selection of $\lambda$ is not always easy and certain. In presence of uncertainty in noise and at low SNR the threshold selection becomes more of an optimization problem. According to Lai et al. (2015), in this paper minimization of error probability $\left(P_{E}=P_{M D}+P_{F A}\right)$ is taken as the objective function to derive the optimal threshold. The detection parameters of Eqs. (6) and (7) in terms of noise uncertainty $\rho$ are used to compute the $\mathrm{P}_{\mathrm{E}}$. To obtain optimal threshold value, the error rate with respect to threshold, $\frac{\partial P_{E}}{\partial}=0 . \quad$ Taking $\quad Q(y)=\frac{1}{2} \operatorname{erfc}\left(\frac{y}{\sqrt{2}}\right) \quad$ where $\frac{\partial \operatorname{erfc}\left(\frac{y-a}{b}\right)}{\partial y}=-\frac{2 e^{-\frac{(y-a)^{2}}{b^{2}}}}{b \sqrt{\pi}}$, the equation for threshold optimization can be re-written in terms of $\rho$ as given in Avila and Thenmozhi (2015) and represented in (15): 
$\frac{\partial P_{E}}{\partial \lambda}=\frac{e^{-\frac{\left(\lambda-N(1+S N R) \rho \sigma_{n}^{2}\right)^{2}}{2 N(1+2 S N R) \rho^{2} \sigma_{n}^{4}}}}{\sqrt{2 \pi N(1+2 S N R)} \rho \sigma_{n}^{2}}-\frac{e^{-\frac{\left(\lambda-N \rho \sigma_{n}^{2}\right)^{2}}{2 N \rho^{2} \sigma_{n}^{4}}}}{\sqrt{2 \pi N} \rho \sigma_{n}^{2}}=0$

Since the optimization of threshold is being performed for the uncertainty zone where $\rho>1$ and SNR $<<1$ (low SNR), the term $(1+2 S N R) \approx 1$ in the denominator of Eq. (15), resulting in the following quadratic equation after taking natural logarithm $(\ln )$ on both sides:

$\lambda^{2}-N \sigma_{n}^{2} \rho \lambda-N^{2} \sigma_{n}^{2} \rho^{2} S N R=0$

The possible roots of Eq. (14) are: $=\frac{N \sigma_{n}^{2} \rho \pm \sqrt{N^{2} \sigma_{n}^{4} \rho^{2} S N R}}{2}$,

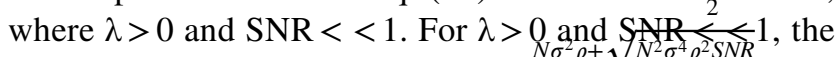
selected decision threshold is: $\lambda=\frac{N \sigma_{n}^{2} \rho+\sqrt{N^{2} \sigma_{n}^{4} \rho^{2} S N R}}{2}$. The optimal value of threshold can be approximated as:

$\lambda^{*}=\frac{N \sigma_{n}^{2} \rho+\sqrt{N^{2} \sigma_{n}^{4} \rho^{2}}}{2}$

$\lambda^{*}=N \sigma_{n}^{2} \rho$

Thus, to avoid sensing failure in the region of confusion in presence of noise power fluctuations, the threshold is optimized using Eq. (17). This signifies that there exists one such decision threshold for energy detection-based spectrum sensing method which can yield minimal error without disturbing the PU's activity at very low SNR. The Eq. (17) is a closed form expression for most suitable and optimal threshold calculation. To maintain a smaller number of samples $\mathrm{N}$ and keep $\mathrm{P}_{\mathrm{MD}}$ and $\mathrm{P}_{\mathrm{FA}}$ within acceptable limits, threshold $\lambda$ can be adjusted in uncertain noisy environment using the expression in (17). Further, taking logarithm and multiplying by 10 on both sides of Eq. (17), we get:

$10 \log _{10} \lambda^{*}=10 \log _{10} N+10 \log _{10} \sigma_{n}^{2}+10 \log _{10} \rho$

The threshold $\lambda^{*}$ in Eq. (18) is proposed as the optimal threshold for spectrum sensing in the region of confusion at very low SNR in presence of $\rho$ to achieve minimal probability of error. The uncertainty in noise is defined as $10 \log _{10} \rho$ $(\mathrm{dB})$ and the sample complexity is $10 \log _{10} N$ for better presentation of the obtain results. Keeping N small, $P_{F A} \leq 10 \%$, the threshold can be made adaptive using Eqs. (12) and (13) to obtain desirable results in terms of higher $P_{D}$ and acceptable sensing time $\tau$. The next section discusses the simulation results and analyse the proposed model with the existing methods of spectrum sensing using Energy detection.

\section{Simulation results}

To evaluate the adeptness of the proposed model, MATLAB based simulations were performed and analysed. The proposed adaptive double threshold method (ED_ADT) with optimal thresholding is compared with conventional single and double threshold energy detection (ED, ED_DT) methods with no noise uncertainty and under noise uncertainty for validation. According to the WRAN (Wireless Regional Area Network) standard IEEE 802.22 based on Cognitive radio technology; detection of $\mathrm{PU}$ signals is required to be done at a very low SNR of about $-20 \mathrm{~dB}$ and receiver sensitivity as low as $-116 \mathrm{dBm}$ (Cordeiro et al., 2006). Therefore, the focus of this work too is on the low SNR region, and for simulation purpose it is considered that the SNR varies from -20 to $5 \mathrm{~dB}$; the noise is AWGN with mean $\mu=0$ and $\sigma_{n}^{2}=1$, noise uncertainty is taken as $1<\rho<1.06$; the number of samples $10<\mathrm{N}<5000$ from Madhunala and Rallapalli (2020), Atapattu et al. (2011).

The simulated plot in Fig. 4 shows the damaging consequence of noise uncertainty on the detection probability at low SNR for a conventional single threshold-based Energy Detection (ED) method with varying values of $\mathrm{N}$. It is noted with small uncertainty in noise the detection probability reduces at low SNR and it worsens as the number of samples reduces $(\rho=1.02)$. However, the detection probability exhibits robustness to uncertainty at higher SNR.

The comparison between the existing double thresholdbased detection ED_DT and proposed adaptive threshold based ED_ADT in terms of Receiver Operating Characteristics (ROC) curve $\left(\mathrm{P}_{\mathrm{D}} \mathrm{Vs}_{\mathrm{FA}}\right)$ with $\mathrm{N}=2000, \mathrm{SNR}=-10 \mathrm{~dB}$ and $\rho=1.02,1.04$ is presented in Fig. 5. It depicts that the ED_ADT performs better than the ED_DT method with noise uncertainty $\rho=1.02,1.04$ by 39.63 and $27.22 \%$ respectively. The existing double threshold methods as discussed in Eqs. (10) and (11) of Sect. 4 has been abbreviated as ED_DT in the simulated results. It can be observed in Fig. 5 that, at $\rho=1.02,1.04$, the ED_DT method offers lower detection probability $\mathrm{P}_{\mathrm{D}}$ as compared to the proposed adaptive double threshold-based technique (ED_ADT) with the use

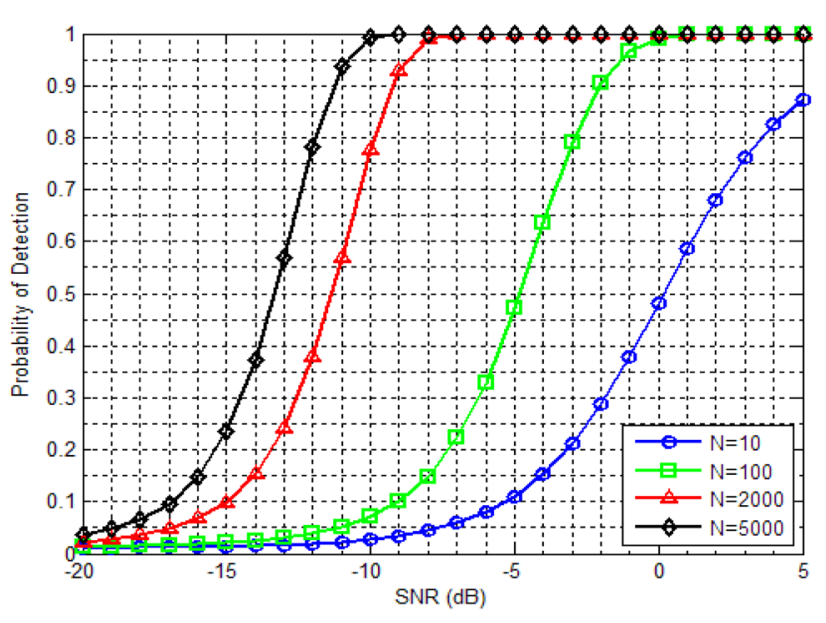

Fig. 4 Effect of Noise uncertainty factor on detection probability 


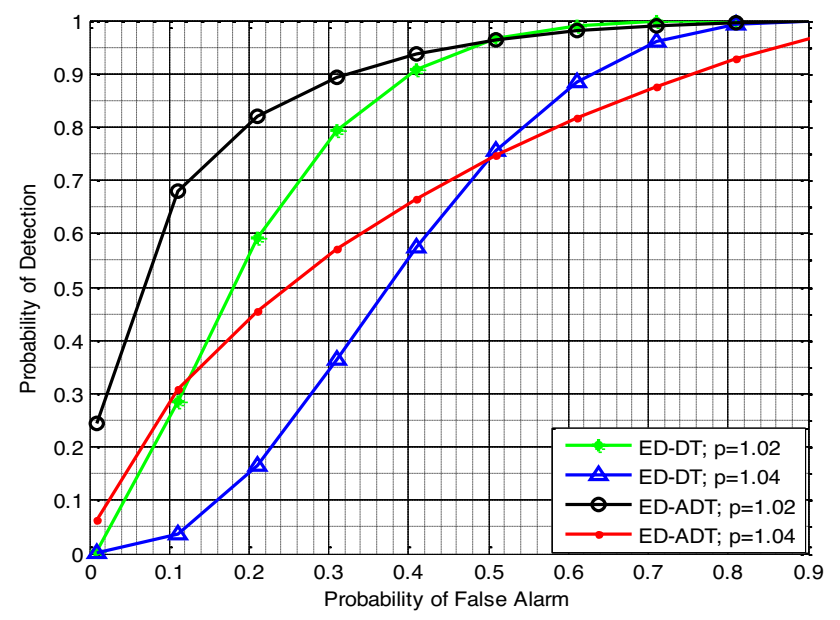

Fig. 5 ROC $\left(\mathrm{P}_{\mathrm{D}}\right.$ Vs $\left.\mathrm{P}_{\mathrm{FA}}\right)$ for varying noise uncertainty factor $\rho=1.02$; 1.04

of Eqs. (12) and (13). The unique feature to be pointed out here is that the ED_ADT gives increased $\mathrm{P}_{\mathrm{D}}$ when $\mathrm{P}_{\mathrm{FA}}<50 \%$ (low) especially. Moreover, when noise uncertainty factor increases, the $\mathrm{P}_{\mathrm{D}}$ in ED_DT reduces but ED_ADT exhibits robustness against the uncertainty.

The simulated result in Fig. 6 shows the probability of error $\left(\mathrm{P}_{\mathrm{E}}\right)$ with respect to $\mathrm{SNR}(\mathrm{dB})$ for the proposed model based on adaptive thresholds (ED_ADT). It clearly shows that the error probability reduces only under uncertain noise $(\rho>1)$ at low SNR values (SNR $<-5 \mathrm{~dB}$ ), where sensing failure is likely to occur. When noise uncertainty considered is 1.04 , the probability of error increases for existing double threshold and conventional single threshold ED_DT and ED methods. It can be observed from the obtained plot that error rate reduces at higher SNR values with the use of proposed

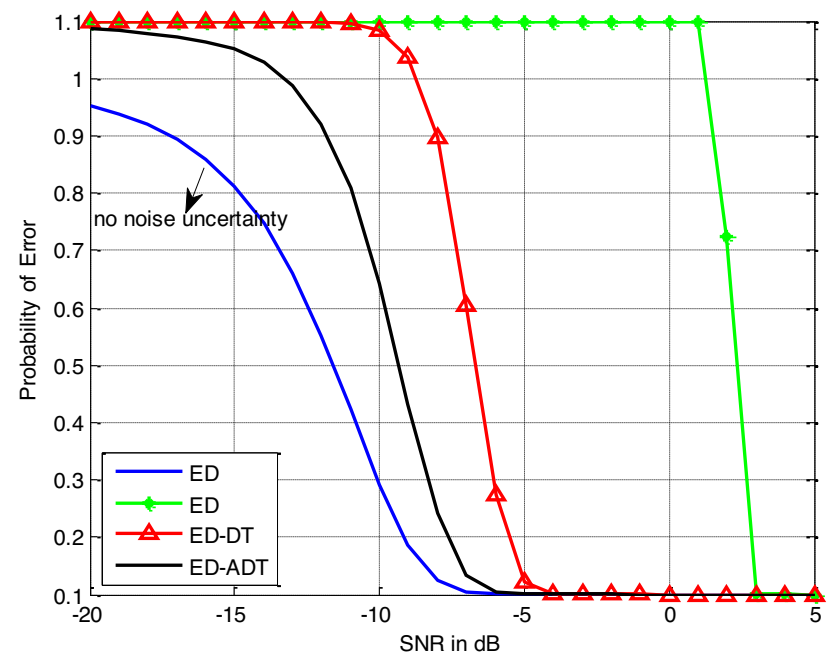

Fig. 6 Probability of Error Vs SNR method ED_ADT. A reduction of about $30 \%$ in error rate is observed using ED_ADT at SNR $=-10 \mathrm{~dB}$ as compared to ED_DT. Thus, the two novel adaptive thresholds reduce the risk of missed detection or false alarm as it reduces the over all error probability.

Furthermore, the error probability is reduced by using optimal threshold as proposed in Sect. 3B. For simulation purpose, the normalized threshold is taken as $\lambda^{*}=\lambda /$ Ns, i.e., the threshold is normalized by the number of samples (Lai et al. 2015). The Receiver Operator Characteristic curves were obtained at different values of $0.95 \leq \lambda^{*} \leq 1.50$ for $\mathrm{SNR}=-10 \mathrm{~dB}$ and $-5 \mathrm{~dB}$ when $\mathrm{N}=2000$. It is shown in Fig. 7 that the total error rate $\mathrm{P}_{\mathrm{E}}$ is reduced to a minimum value at optimal value of $\lambda^{*}$.

The $\mathrm{P}_{\mathrm{E}}$ versus normalized threshold for AWGN channel in presence of noise uncertainty $\rho=1.02,1.04,1.06$ is depicted in Fig. 7 for $\mathrm{SNR}=-15 \mathrm{~dB}$ and $\mathrm{SNR}=-10 \mathrm{~dB}$ at $\mathrm{N}=2000$. The optimal threshold at $\rho=1.02$ and SNR $=-10 \mathrm{~dB}$ is $\lambda^{*}=0.78$ i.e. $-34 \mathrm{~dB}$, and for $\mathrm{SNR}=-15 \mathrm{~dB}, \rho=1.06$, $\lambda^{*}=0.84$ i.e. $-32 \mathrm{~dB}$. It denotes that the value of optimal threshold and error probability increases with the increasing uncertainty in noise. By increasing the number of samples $\mathrm{N}$ at $\mathrm{SNR}=-15 \mathrm{~dB}$ and $\rho=1.04$, the probability of error can be further reduced as shown in Fig. 8. This reduction justifies the proposed Eq. (17) and validates the minimization achieved at optimal threshold with increasing sample number. It clearly marks that minimal error rate $P_{E}=0.85,0.5,0.3$ is attained at $\lambda^{*}=0.77$ at $\mathrm{N}=500,2000$ and 5000 respectively. Thus, desirable sensing performance can be achieved by adjusting $\mathrm{N}$ at higher noise uncertainty and lower SNR by using the proposed optimal threshold Eq. (16). The plot in Fig. 9 presents the sample complexity $10 \log _{10} \mathrm{~N}$ with respect to SNR for ED, ED_DT and

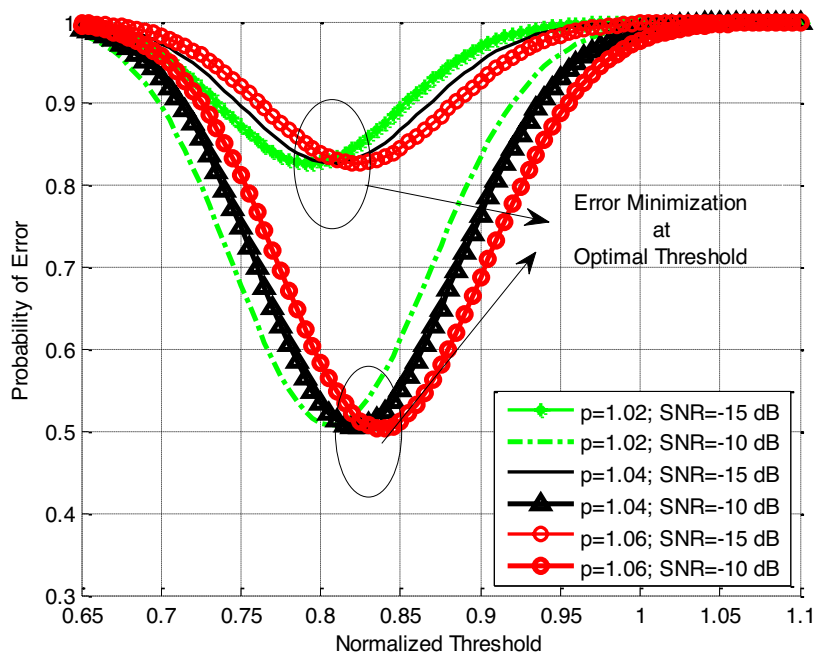

Fig. 7 Probability of Error versus Threshold for varying noise uncertainty $\rho$ 


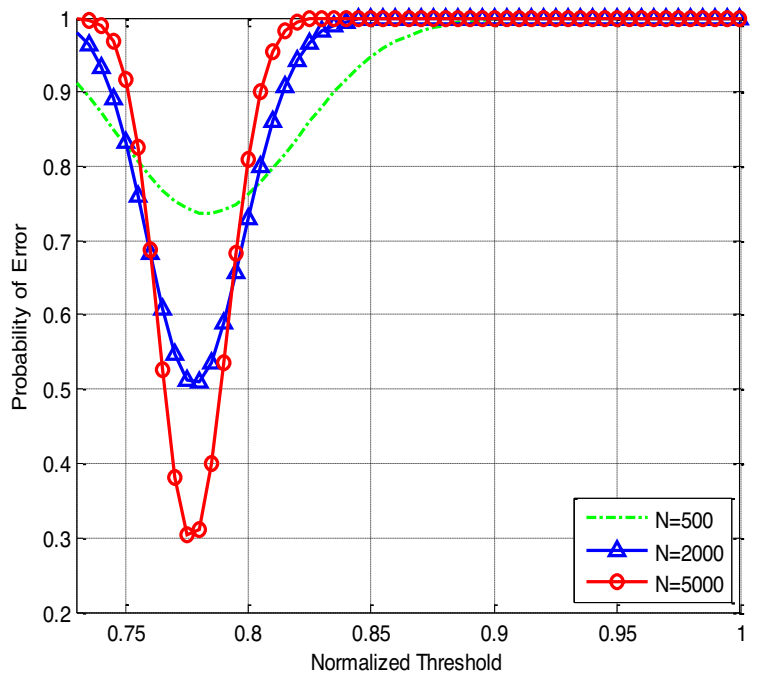

Fig. 8 Probability of Error versus Normalized Threshold for varying number of samples

ED_ADT. Sensing failure has been marked to signify the sharp rise in $\mathrm{N}(N \rightarrow \infty)$ between $(-14<\mathrm{SNR}<-12 \mathrm{~dB})$ when $\rho>1$ (1.02) for ED and ED_DT methods. This sensing failure is avoided using proposed ED_ADT method with a significant drop in $\mathrm{N}$ at $\mathrm{SNR}=-12 \mathrm{~dB}$. Finally, the result in Fig. 10 represents sample complexity with respect to SNR at increasing values of $\rho$. The detection failure is overcome by increasing the dynamic threshold parameter $\rho$ ' with respect to the increasing uncertainty $\rho$. Though the sample number $\mathrm{N}$ increases when the uncertainty in noise becomes higher, but detection failure is overcome in all the cases $(\rho=1.02,1.04,1.06)$. By manipulating the value of $\rho^{\prime}$ $\left(\rho^{\prime}>\rho\right)$, an abrupt increment in $\mathrm{N}$ is eschewed and sensing

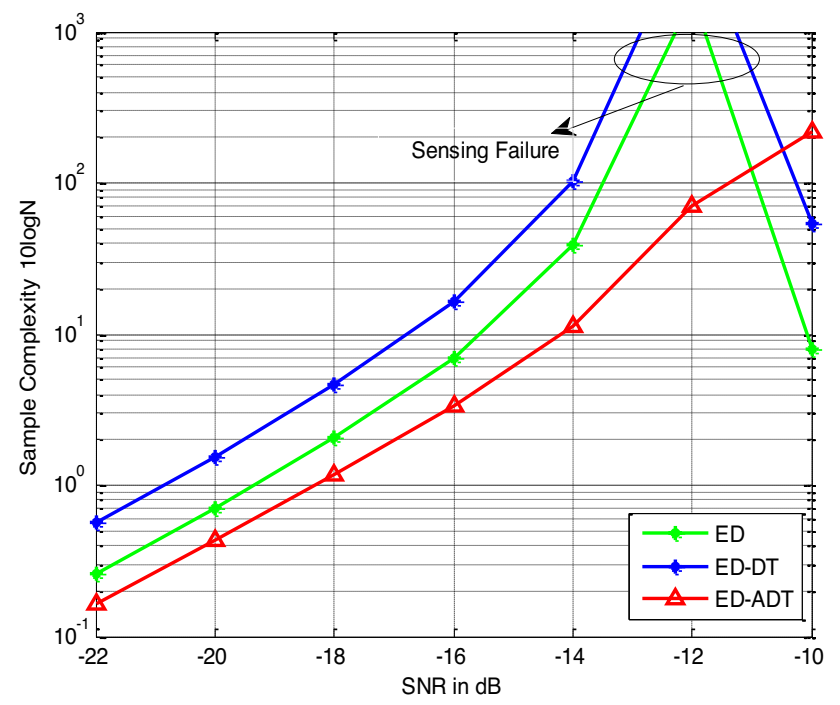

Fig. 9 Sample Complexity versus SNR

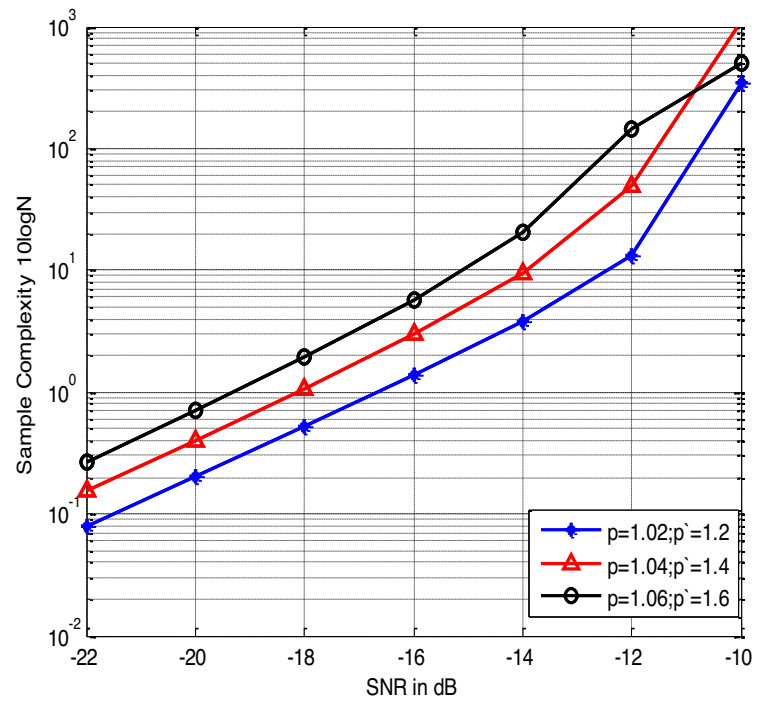

Fig. 10 Sample Number versus SNR for varying $\rho$ and $\rho$ ' at $\mathrm{SNR}=-10 \mathrm{~dB}$

is made successful. This was the reason why cognitive radio sensing is more focused on achieving smaller $\mathrm{N}$ or sensing time for detection at low SNR. Therefore, the proposed model ED_ADT outperforms ED_DT substantially.

\section{Conclusion and future scope}

A mathematical approach to compute optimal threshold to achieve minimum error rate for spectrum sensing in cognitive radio systems in presence of noise uncertainty using energy detection has been proposed. The uncertainty in noise is considered and its effect on $\mathrm{P}_{\mathrm{D}}$ and $\mathrm{P}_{\mathrm{E}}$ has been studied and analyzed. It can be summarized that the proposed model can be utilized for estimation of adaptive double thresholds for low SNR region, and optimal threshold for confused region. Such an approach is of significant use for spectrum sensing in a low SNR scenario to avoid the sensing capability-sensing error tradeoff issue. The simulated results for ED_ADT validate the novel findings and propose to improve the sensing performance $\left(\mathrm{P}_{\mathrm{D}}\right)$ by $39.63 \%$ and $27.22 \%$ at low $\mathrm{SNR}=-10 \mathrm{~dB}$ and $-15 \mathrm{~dB}$ with noise uncertainty factor $\rho=1.02,1.04$ respectively in comparison to existing double threshold method ED_DT. Similarly, probability of error is reduced at low SNR $(<-5 \mathrm{~dB})$ in presence of uncertainty in noise using the proposed model with adaptive double threshold ED_ADT. The derived expression for optimal threshold reduces the error rate to a minimum value of 0.3 at an optimal threshold for uncertain noise at $\mathrm{SNR}=10 \mathrm{~dB}$. A noteworthy reduction in number of samples with respect to SNR and noise level uncertainty is also reported that highlights the superiority of the proposed model. Hence, it is inferred 
from the work that the proposed scheme of spectrum sensing (ED_ADT) increases the detection probability, avoids sensing failure and reduces the chances of missed detection and false alarm for lower SNR with uncertain noise. The optimal threshold from the suggested equation minimizes the error rate even at very low SNR values and increasing uncertainty in noise.

Furthermore, the results presented in this work can be augmented with the use of other optimization techniques like particle swarm optimization, along with the proposed adaptive double thresholds-based sensing. This works focuses on detection probability and error rate only, however, a study on energy efficiency and throughput of the system could also be a future prospect.

\section{References}

Alink MSO, Kokkeler AB, Klumperink EA, Smit GJ, Nauta B (2011) Lowering the SNR wall for energy detection using cross-correlation. IEEE Trans Veh Technol 60(8):3748-3757

Anandakumar H, Umamaheswari K (2017) An efficient optimized handover in cognitive radio networks using cooperative spectrum sensing. Intell Autom Soft Comput, pp 1-8

Arjoune Y, Kaabouch N (2019) A comprehensive survey on spectrum sensing in cognitive radio networks: Recent advances, new challenges, and future research directions. Sensors 19(1):126

Atapattu S, Tellambura C, Jiang H (2011) Spectrum sensing via energy detector in low SNR. In: 2011 IEEE International Conference on Communications (ICC), pp 1-5

Avila J, Thenmozhi K (2015) Adaptive double threshold with multiple energy detection technique in cognitive radio. Res J Appl Sci Eng Technol 10(11):1336-1342

Bagheri A, Ebrahimzadeh A, Najimi M (2020) Energy-efficient sensor selection for multi-channel cooperative spectrum sensing based on game theory. J Ambient Intell Human Comput 12(10):9363-9374

Bhowmick A, Chandra A, Roy SD, Kundu S (2015) Double thresholdbased cooperative spectrum sensing for a cognitive radio network with improved energy detectors. IET Commun 9(18):2216-2226

Cordeiro C, Challpali K, Birru D (2006) IEEE 802.22: an introduction to the first wireless standard based on cognitive radios. J Commun $1: 38-47$

Haykin S (2005) Cognitive radio: brain-empowered wireless communications. IEEE J Sel Areas Commun 23(2):201-220

Hosseini SAT, Amindavar H, Ritcey JA (2010) A new cyclostationary spectrum sensing approach in cognitive radio. In: 2010 Signal Processing Advances in Wireless Communications (SPAWC), pp 1-4

Lai M, Peng S, Yang X, Zhou L (2015) Fast two-step energy detection for spectrum sensing. J Electric Comput Eng 2015:591627(1)-591627(6)

Letaief KB, Zhang W (2009) Cooperative communications for cognitive radio networks. Proc IEEE 97(5):878-893

Ling X, Wu B, Wen H, Ho PH, Bao Z, Pan L (2012) Adaptive threshold control for energy detection based spectrum sensing in cognitive radios. IEEE Wirel Commun Lett 1(5):448-451

Lutu A, Perino D, Bagnulo M, Frias-Martinez E, Khangosstar J (2020) A characterization of the COVID-19 pandemic impact on a mobile network operator traffic. In : 2020 ACM Internet Measurement Conference, pp 19-33

Mabrook MM, Taha HA, Hussein AI (2020) Cooperative spectrum sensing optimization based adaptive neuro-fuzzy inference system
(ANFIS) in cognitive radio networks. J Ambient Intell Human Comput, pp 1-9

Madhunala S, Rallapalli H (2020) Throughput and spectrum sensing trade-off by incorporating self-interference suppression for full duplex cognitive radio. In: Advances in Decision Sciences, Image Processing, Security and Computer Vision, pp 130-138

Mamidi R, Sundru A (2018) Throughput analysis in proposed cooperative spectrum sensing network with an improved energy detector scheme over Rayleigh fading channel. AEU-Int J of Electron Commun 83:416-426

Mariani A, Giorgetti A, Chiani M (2011) SNR wall for energy detection with noise power estimation. In: 2011 IEEE International Conference on Communications (ICC), pp 1-6

McHenry M (2005) NSF spectrum occupancy measurements project summary. Tech. Rep.

Mitola J, Maguire GQ (1999) Cognitive radio: making software radios more personal. IEEE Pers Commun 6(4):13-18

Na JC (2017) Optimization in cooperative spectrum sensing. Asia-Pac J Converg Res Interchange 3(1):19-31

Shukla S, Rao AK, Srivastava N (2016) A survey on energy detection schemes in cognitive radios. In: 2016 International Conference on emerging trends in electrical electronics \& sustainable energy systems (ICETEESES), pp 223-228

Sobron I, Diniz PS, Martins WA, Velez M (2015) Energy detection technique for adaptive spectrum sensing. IEEE Trans Commun 63(3):617-627

Suneel AS, Shiyamala S (2021) Peak detection based energy detection of a spectrum under rayleigh fading noise environment. J Ambient Intell Human Comput 12(3):4237-4245

Tandra R, Sahai A (2008) SNR walls for signal detection. IEEE J Sel Top Signal Process 2(1):4-17

Tech. Rep. (2002) Spectrum policy task force report, 02-135

Urkowitz H (1967) Energy detection of unknown deterministic signals. Proc IEEE 55(4):523-531

Vadivelu R, Sankaranarayanan K, Vijayakumari V (2014) Matched Filter based Spectrum sensing for cognitive radio at low signal to noise ratio. J Theoret Appl Info Technol 62(1):107-113

Valenta V, Marsalek R, Baudoin G, Villegas M, Suarez M, Robert F (2010) Survey on spectrum utilization in Europe: measurements, analyses and observations. In: 2010 International Conference on Cognitive Radio Oriented Wireless Networks and Communications (ICST), pp 1-5

Verma P, Singh B (2015) Simulation study of double threshold energy detection method for cognitive radios. In: Signal Processing and Integrated Networks (SPIN), pp 232-236

Verma P, Singh B (2016) Overcoming sensing failure problem in double threshold based cooperative spectrum sensing. Optik 127(10):4200-4204

Xie J, Chen J (2012) An adaptive double-threshold spectrum sensing algorithm under noise uncertainty. In: Computer and Information Technology (CIT), pp 824-827

Yu G, Long C, Xiang M (2012) A novel spectrum detection scheme based on dynamic threshold in cognitive radio systems. Res J Appl Sci Eng Technol 4(21):4245-4251

Zhu J, Cao H, Zhang J, Zhou Z, Qi Z (2020) Novel double threshold based energy detector for spectrum sensing, signal and information processing, networking and computers. Lect Notes Electric Eng $628: 46$

Publisher's Note Springer Nature remains neutral with regard to jurisdictional claims in published maps and institutional affiliations. 